Matyszewska, Dorota; Bilewicz, Renata; Su, ZhangFei; Leitch, J. Jay; Lipkowski, Jacek

"PM-IRRAS studies of DMPC bilayers supported on $\mathrm{Au}(111)$ electrodes modified with hydrophilic monolayers of thioglucose." Langmuir, 2016,32,1791-1798.

\title{
PM-IRRAS studies of DMPC bilayers supported on Au(111) electrodes modified with hydrophilic monolayers of thioglucose
}

Dorota Matyszewska ${ }^{1 *}$, Renata Bilewicz ${ }^{2}$, ZhangFei $\mathrm{Su}^{3}$, Fatemah Abbasi ${ }^{3}$, J. Jay Leitch ${ }^{3}$, and Jacek Lipkowski ${ }^{3}$

${ }^{1}$ Faculty of Chemistry, Biological and Chemical Research Centre, University of Warsaw, Żwirki i Wigury 101, 02089 Warsaw, Poland

${ }^{2}$ Faculty of Chemistry, University of Warsaw, ul. Pasteura 1, 02093 Warsaw, Poland

${ }^{3}$ Department of Chemistry, University of Guelph, Guelph, Ontario, N1G 2W1, Canada

\begin{abstract}

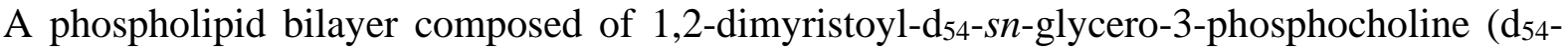
DMPC) was deposited onto the $\mathrm{Au}(111)$ electrode modified with a self-assembled monolayer of 1-thio- $\beta$-D-glucose $(\beta$-Tg) via the Langmuir-Blodgett and Langmuir-Schaefer (LB-LS) techniques. Polarization modulation infrared reflection absorption spectroscopy (PM-IRRAS) measurements were used to characterize structural and orientational changes in this model biological membrane in the presence of hydrophilic layer on the surface of the electrode. The results of the spectroscopic measurements showed that the tilt angle of acyl chains obtained for deuterated DMPC bilayers supported on the $\beta$-Tg-modified gold is significantly lower than that reported previously for DMPC bilayers deposited directly on $\mathrm{Au}(111)$ electrodes. Moreover, tilt angles of $\sim 18^{\circ}$ were obtained for $\mathrm{d}_{54}$-DMPC bilayers on $\beta$-Tg self-assembled monolayers (SAMs) at positive potentials, which are similar to the values calculated for h-
\end{abstract}


DMPC deposited on bare gold in the desorbed state and to the tilt angles observed for a stack of hydrated DMPC bilayers. This data confirms that the $\beta$-thioglucose SAM promotes the formation of a water cushion that separates the phospholipid bilayer from the metal surface. As a result, the DMPC polar heads are not in direct contact with the electrode and can adopt a zigzag configuration, which strengthens the chain-chain interactions and allows for an overall decrease in the tilt of the acyl chains. These novel supported model membranes may be especially useful in studies pertaining to the incorporation of peptides and proteins into phospholipid bilayers.

Key-words: 1-thio- $\beta$-D-glucose $(\beta$-Tg), 1,2-dimyristoyl-d54-sn-glycero-3-phosphocholine ( $\mathrm{d}_{54}$-DMPC), model lipid membrane, polarization modulation infrared reflection absorption spectroscopy (PM-IRRAS). 


\section{Introduction}

The Langmuir technique allows for the formation of amphiphilic monolayers at the airwater interface and therefore has been widely used to study the interactions of a wide variety of different species, such as drugs, toxins, peptides, proteins or other biologically important molecules, with simplified biological model membranes. ${ }^{1,2,3,4,5}$ These molecule-membrane interactions are monitored at the air-water interface by measuring changes in the collapse pressure, compression modulus and area per molecule. However, these measurements are typically limited to monolayer films and do not provide molecular level information about the effects that these species have on the individual molecules within the monolayer. In order to better mimic a natural biological membrane, the combination of Langmuir-Blodgett and Langmuir-Schaefer techniques may be employed to prepare supported bilayer lipid membranes (sBLMs). Typically, a single type of phospholipid, such as DMPC, is used to assemble the biomimetic layers onto the gold surface. ${ }^{6,7}$ Alternatively, cholesterol may be introduced into these films to improve the elastic properties of the membrane and more closely resemble the true composition of biological cell membranes. ${ }^{8,9}$ In addition, the transfer of the bilayers onto the surface of the electrode enables the application of various electrochemical, microscopic and spectroscopic techniques to gain a molecular level description of the sBLM, including the morphology, orientation, conformation and organization of the molecules within the bilayer. ${ }^{10,11,12}$ Such models have been successfully used to study the incorporation of peptides or interactions with toxins. ${ }^{13,14,15,16}$

After transferring the phospholipid bilayer onto the solid support, the head groups of the bottom layer are directly in contact with the metal surface, which influences the overall structure of the bilayer. This issue becomes especially important in studies involving the incorporation of transmembrane proteins into the supported membranes because contact with metal may lead to denaturation or changes in the functionality of the proteins. Therefore, 
different models geared towards the separation of the bilayer from the metal surface have been proposed in the literature. In tethered bilayer lipid membranes (tBLMs), the lipid bilayer is covalently coupled to the solid support via a flexible spacer moiety. ${ }^{17}$ For this purpose, different types of compounds containing thiol- or disulfide groups, which can be chemisorbed on the gold electrode, are frequently used to form the inner (or cystosolic) leaflet of the bilayer. Common examples of these tethering compounds include thiopeptides or thiolipids. ${ }^{18,}$ 19 The outer (or extracellular) leaflet of the bilayer can then be formed by depositing a phospholipid monolayer on top of the self-assembled inner leaflet via vesicle spreading or a Langmuir-Schaefer deposition.

Another type of model system where the bilayer is not directly in contact with metal surface is a floating bilayer lipid membrane (fBLM). The mobility of this bilayer is enhanced by separating the membrane from the solid substrate by a water-rich domain and absence of a chemically attached inner leaflet. ${ }^{20}$ In fBLMs, the biomimetic bilayer is often placed on the surface of another lipid bilayer that has been previously deposited onto the solid support. These specific fBLMs are also referred to as stacked lipid bilayers. ${ }^{21}$ Alternatively, lipid bilayers can be separated from the substrate by a polyethylene glycol (PEG) spacer. $^{22,}{ }^{23} \mathrm{In}$ order to provide ample separation, the head group of the molecules within the inner leaflet of a phospholipid bilayer should be modified with the polymer. Such model systems have proved to be robust and reproducible and have been characterized by a number of different experimental techniques including electrochemistry, microscopy and spectroscopy. In addition, these designs have been successful in the study of peptide ion channels such as gramicidin. ${ }^{24}$ A further fBLM design employs the addition of monosialoganglioside (GM1) into the inner leaflet of the bilayer in order to promote the formation of a water reservoir between the bilayer and solid substrate. ${ }^{25}$ Kycia et al. showed that modifying the $\mathrm{Au}(111)$ electrode with a self-assembled monolayer (SAM) of 1-thio- $\beta$-D-glucose $(\beta-\mathrm{Tg})$ produced a 
stable, hydrophilic layer, which was well characterized by electrochemical, microscopic and spectroscopic methods. ${ }^{26,27}$ These $\beta$-Tg SAMs separate the bilayer from the metal surface by providing the necessary water cushion and have been successful in the preparation of floating bilayer lipid membranes. ${ }^{25}$

The floating bilayer in the paper by Kycia et $\mathrm{al}^{25}$ was designed to study colicin E1 channel Peptide. To allow protein insertion and protect against denaturation, the membrane has to be separated from the gold surface by a 1-2-nm-thick water-rich layer. However, investigations of short chain antimicrobial peptides such as alamethicin or gramicidin, with chain length less than the hydrophobic thickness of the bilayer do not require such a thick water space between the metal and the bilayer. To avoid phase separation in the multicomponent mixture of lipids it is advantageous to investigate these peptides in a single component bilayer. We will show that the modification of the gold surface with thioglucose allows for better packing of the lipids which in consequence may prevent stress in the peptide helix such as that observed when gramicidin is incorporated into the bilayer that is in a direct contact with the metal. ${ }^{28}$

In this work, PM-IRRAS was employed to study the orientation and conformation of the acyl chains in DMPC bilayers deposited on gold electrodes modified with a self-assembled layer of $\beta$-thioglucose. To achieve this goal, DMPC bilayers, consisting of deuterium substituted molecules, were deposited onto $\beta$-Tg-modified $\mathrm{Au}(111)$ electrode surfaces using the Langmuir-Blodgett and Langmuir-Schaefer (LB-LS) techniques. This approach provides a means of separating the IR bands of the phospholipid bilayer from spectral interferences due to the presence of underlying hydrogen substituted $\beta$-Tg SAM. The objective of this study was to determine the packing of the phospholipid acyl chains in this floating bilayer and confirm that the monolayer of the hydrophilic SAM provides a water rich environment on the inner side of the floating bilayer. 


\section{Experimental}

Reagents and Materials. 1,2-dimyristoyl-d $\mathrm{d}_{54}-s n$-glycero-3-phosphocholine ( $\mathrm{d}_{54}$-DMPC) and 1-thio- $\beta$-D-glucose sodium salt $(\beta-\mathrm{Tg})$ were purchased from Avanti Polar Lipids and Sigma-Aldrich, respectively. $\mathrm{d}_{54}$-DMPC solution was prepared by dissolving the lipid in HPLC-grade chloroform (Sigma-Aldrich) to obtain a concentration of $1 \mathrm{mg} / \mathrm{ml}$, while $\beta$-Tg solution $(2 \mathrm{mM})$ was prepared by dissolving in methanol (Sigma-Aldrich). The electrolyte solution for the PM-IRRAS experiments was prepared from sodium fluoride (Sigma-Aldrich) dissolved in ultrapure Milli-Q UV plus water (resistivity > $18.2 \mathrm{M} \Omega \mathrm{cm}$ ) (Millipore, Bedford, MA). Prior to use, the NaF powder was placed in an UV ozone chamber (UVO cleaner, Jelight, Irvine, CA.) for 20 min to oxidize and remove any organic impurities.

Prior to the experiments, all glassware was cleaned in a hot mixed acid bath (1:3 $\mathrm{HNO}_{3} / \mathrm{H}_{2} \mathrm{SO}_{4}$ ) for approximately 45 min and thoroughly rinsed using a Milli-Q water. The Teflon parts of the spectroelectrochemical cell were cleaned in piranha solution and thoroughly rinsed with Milli-Q water. The single crystal Au(111) electrodes (disc type), which were grown, oriented, and cut in our laboratory, were used as the working electrodes in the electrochemical and PM-IRRAS experiments. The electrical contact was provided by spot-welding of the gold wire to the backside of the electrode. The Au(111) electrodes were cleaned by consecutive flame-annealing, cooling and rinsing with copious amounts of Mill-Q water at the beginning of each experiment. Additionally, a $\mathrm{Ag} / \mathrm{AgCl}$ (sat. $\mathrm{KCl}$ ) and $\mathrm{Pt}$ wire were used as the reference and counter electrodes, respectively.

Bilayer deposition. Prior to the bilayer deposition, the $\mathrm{Au}(111)$ electrode was modified by a self-assembled layer of $\beta$-thioglucose. The self- assembly was accomplished by immersing the cleaned electrode into the methanolic solution of $\beta$-Tg overnight. The 
electrode was then rinsed with copious amounts of methanol to remove any physically adsorbed $\beta$-Tg molecules and next with Milli-Q water to remove the residual methanol.

The deuterated DMPC bilayer was deposited onto the $\beta$-Tg covered $\mathrm{Au}(111)$ electrode using the combination of Langmuir-Blodgett (LB) and Langmuir-Schaefer (LS) methods. A KSV-Nima Mini-Micro Langmuir trough with two hydrophilic barriers and a Wilhelmy balance equipped with a Wilhelmy plate (fabricated from filter paper) controlled by KSV LB5000 software was used to transfer the phospholipid monolayers. The water subphase, used for both the LB and LS transfers, was heated to a temperature of $30^{\circ} \mathrm{C}$ to improve the homogeneity and fluidity of the film. ${ }^{29}$ The bottom leaflet composed of $\mathrm{d}_{54}$-DMPC was spread at the air-water interface using a Hamilton microsyringe. After 15 min of solvent evaporation, the layer was slowly compressed to a surface pressure of $30 \mathrm{mN} / \mathrm{m}$ and transferred onto the $\beta$-Tg covered $\mathrm{Au}(111)$ electrode placed in the Teflon holder allowing for the proper orientation of the single crystal electrode by vertical withdrawal at a speed of 12 $\mathrm{mm} / \mathrm{min}$ in order to obtain the transfer ratio of $1.0 \pm 0.1$. The electrode was allowed to dry for $1 \mathrm{~h}$ before the depositing the top leaflet via the LS method. The electrode was horizontally brought into contact with the $\mathrm{d}_{54}-\mathrm{DMPC}$ monolayer that was compressed to $30 \mathrm{mN} / \mathrm{m}$ at the air-water interface. The electrode was then slowly lifted upwards until it broke the contact with the subphase and was allowed to dry for $1 \mathrm{~h}$ before inserting into the spectroelectrochemical cell.

Differential Capacitance Measurements. The differential capacities were calculated from the in-phase and out-of-phase components of the ac signal assuming a simple series RC equivalent circuit. A computer controlled system, consisting of a HEKA PG 590 potentiostat/galvanostat and a lock-in amplifier (EG\&G Instruments $7265 \mathrm{DSP}$ ), was used to perform these experiments. All data were acquired via a plug-in acquisition board (National 
Instruments NI-DAQ BNC-2090) using custom written software generously provided by Professor Dan Bizzotto of the University of British Columbia. The differential capacitance measurements were done in an all glass three-electrode electrochemical cell. The single crystal $\mathrm{Au}(111)$ working electrode and gold wire counter electrode were flame annealed using a Bunsen burner and quenched with Milli-Q ultrapure water to ensure that the electrode surfaces were free from contamination. A silver/silver chloride electrode (SSCE) was used as the reference electrode.

Force distance curve measurements. The force versus distance curve measurements were carried out using V-shape SiN cantilevers with a nominal spring constant of $\sim 0.06 \mathrm{~N} / \mathrm{m}$ (Digital Instruments) mounted onto the Agilent 5500 microscope and the AFMS182 scanner. The tips were exposed to ozone in a UV laminar flow cabinet (Jelight, Irvine, CA) for 30 min prior to use. The force-distance curves were recorded by measuring the deflection of the cantilever versus the position of the sample mounted onto the piezoelectric translator. The original cantilever deflection versus piezo position curves were converted to force curves using software written in-house and the nominal value of the spring constant provided by the supplier. The time needed to record one force curve was $\sim 1.5 \mathrm{~s}$. The force curve measurements were repeated $\sim 100$ times for the purpose of statistical data analysis.

Spectra Collection and Processing. PM-IRRAS experiments were performed using a Nicolet Nexus 870 spectrometer equipped with an external tabletop optical mount, MCT-A detector, photoelastic modulator with a II/ZS50 ZnSe $50 \mathrm{kHz}$ optical head (Hinds Instruments PM-90), and a synchronous sampling demodulator (GWC Instruments). The tabletop optical module (TOM) box was purged for $\sim 12 \mathrm{~h}$ prior to and throughout the duration of the experiment with ultra-dry, $\mathrm{CO}_{2}$-free air, which was provided by a FTIR Purge gas Generator 
(Parker Balston, Haverhill, MA). The spectra were collected at a temperature of $18 \pm 1{ }^{\circ} \mathrm{C}$ (temperature in the laboratory room controlled by air-conditioning) using in-house software, an Omnic macro and a digital-to-analog converter (Omega, Stamford, CT) to control the potentiostat (EG\&G PAR362, Princeton, NJ). A three-electrode electrochemical IR cell with a 1 in. $\mathrm{CaF}_{2}$ equilateral prism (BoXin, Changchun, China) was used as an IR window. Before each experiment, the window was cleaned with methanol and water and placed in the UV ozone chamber for 20 minutes. The spectroelectrochemical cell was assembled and filled with $0.1 \mathrm{M} \mathrm{NaF}$ dissolved in $\mathrm{H}_{2} \mathrm{O}$. The electrolyte solution was then de-aerated by purging with argon for approximately 30 min prior to the experiment. A potential-step program was used to control voltage with an initial applied potential of $0.4 \mathrm{~V}$. The potentials were stepped in the negative direction by $0.1 \mathrm{~V}$ increments until achieving a final potential of $-1.2 \mathrm{~V}$. A total of 6000 scans were collected and averaged at each potential step.

The IR measurements were carried out with the photoelastic modulator (PEM) set to a half-wave retardation at $2150 \mathrm{~cm}^{-1}$, which corresponds to the C-D stretching region. The angle of incidence of the infrared beam was set to $59^{\circ}$ and the electrolyte thickness between the electrode and the prism was $\sim 5 \mu \mathrm{m}$, which provided a large enhancement of the ppolarized light at the electrode surface and good cancellation of the background solution. The thickness of the electrolyte film was calculated by comparing the experimental reflectivity spectrum of the thin layer cell, which is attenuated due to the layer of solution between the electrode and the IR window, to a theoretical reflectivity curve for the thin layer system, which was calculated from optical constants for a three layer Fresnel model consisting of $\mathrm{CaF}_{2} / \mathrm{H}_{2} \mathrm{O} / \mathrm{Au}$, as previously described. ${ }^{30}$

The demodulation technique developed in the Corn laboratory ${ }^{31,32}$ was used in this work. A modified version of a method described by Buffeteau et al. ${ }^{33}$ was used to correct the average intensity $\left(\left[\boldsymbol{I}_{s}(\boldsymbol{\omega})+\boldsymbol{I}_{\boldsymbol{p}}(\boldsymbol{\omega})\right] / \mathbf{2}\right)$ and the intensity difference $\left(\boldsymbol{I}_{\boldsymbol{s}}(\boldsymbol{\omega})-\boldsymbol{I}_{\boldsymbol{p}}(\boldsymbol{\omega})\right)$ for the 
PEM response functions and for the difference in the optical throughputs for $\mathrm{p}$ - and spolarized light. Finally, the measured spectra were background corrected due to the absorption of IR photons in the thin layer cavity. A spline interpolation technique described by Zamlynny et al. ${ }^{34}$ was used for the background correction. The background corrected spectral plots, $\Delta \mathrm{S}$, are proportional to absorbance, $A$, of the adsorbed molecules:

$$
\Delta S=\frac{2\left(I_{s}-I_{p}\right)}{I_{s}+I_{p}} \approx 2.3 \Gamma \varepsilon=2.3 \mathrm{~A}
$$

where $I_{s}$ and $I_{p}$ are the intensities of $\mathrm{s}$ - and p-polarized radiation, $\Gamma$ is the surface concentration of the adsorbed species and $\varepsilon$ is the decimal molar absorption coefficient of the adsorbed species.

\section{Results and discussion}

Initial characterization of the bilayer. The differential capacitance curves and AFM were used to initially characterize the bilayer assembly. Figure 2 shows the differential capacitance curves for the film free electrode, the electrode covered by a monolayer of $\beta-\mathrm{Tg}$ and with the DMPC bilayer deposited on the $\beta$-Tg modified gold surface. The positive limit of these curves $(\sim 0.4 \mathrm{~V}$ vs $\mathrm{Ag} / \mathrm{AgCl})$ is determined by the onset of gold oxide formation. The data show that the monolayer of $\beta-\mathrm{Tg}$ is stable between 0.3 and $-0.6 \mathrm{~V}$ vs $\mathrm{Ag} / \mathrm{AgCl}$. At more negative potentials it is desorbed and its desorption corresponds to a peak seen at $\sim-0.8 \mathrm{~V}$ vs $\mathrm{Ag} / \mathrm{AgCl}$. The deposition of the bilayer is responsible for lowering the capacitance down to $\sim 7 \mathrm{~F} / \mathrm{cm}^{-2}$ in good agreement with the capacitance of the DMPC bilayer deposited directly at the $\mathrm{Au}(111)$ electrode surface. ${ }^{35}$ The low value of the capacitance is maintained between \pm 0.2 $\mathrm{V}$ vs $\mathrm{Ag} / \mathrm{AgCl}$. The capacitance apparently increases outside this range of potentials indicating that it becomes lifted from the electrode surface. At potentials more negative than - 
$0.4 \mathrm{~V}$ vs $\mathrm{Ag} / \mathrm{AgCl}$ the curve recorded for the bilayer covered electrode almost coincides with the curve recorded for $\beta-\mathrm{Tg}$ modified electrode indicating that the lifting of the bilayer is completed. This result is in a good agreement with the behavior of DMPC bilayer deposited directly onto the gold electrode surface..$^{35}$

The force distance curves recorded with the help of the AFM instruments were used to provide direct proof that the combination of the LB-LS techniques leads to the formation of a bilayer at the $\beta$-Tg modified gold surface. Figure 3 shows the representative force distance curve recorded in this experiment. The force-distance curve displays an onset of repulsive interaction between the tip and the film-covered surface at separations of $\sim 10 \mathrm{~nm}$. At separations less than $5 \mathrm{~nm}$, a characteristic discontinuity is observed followed by a vertical section corresponding to the direct interaction of the tip with the gold surface. The "jump-in" distance corresponding to the discontinuity is usually taken as the film thickness. The inset to Figure 3 shows the distribution of the measured film thickness from 100 force curves. The mean value of the Gaussian fitting of the histogram plot is taken as the film thickness and is equal to $5.4 \mathrm{~nm}$. The penetration distance measured from the force-distance curves gives the thickness of the compressed layer, and hence, it is somewhat smaller than the thickness of the film at equilibrium. Following Dufrene et al., the elastic deformation $\delta$ produced by the AFM tip under a known load can be determined from the formula derived from a Hertzian model. ${ }^{36}$ In the present case the correction amounts to $0.25 \mathrm{~nm}$ giving the corrected thickness of $\sim 4.9$ $\mathrm{nm}$. This number is about $0.9 \mathrm{~nm}$ thicker than for the DMPC bilayer deposited directly at the $\mathrm{Au}(111)$ surface consistently with the presence of a monolayer of $\beta$-Tg molecules as a spacer. ${ }^{35}$ In conclusion the AFM experiments confirmed that the bilayer of DMPC is formed at the TG electrode surface.

PM IRRAS experiments. The $\mathrm{C}-\mathrm{H}$ stretching bands in the IR spectra located between 2800 and $3000 \mathrm{~cm}^{-1}$ provide useful information concerning the conformation and orientation 
of the phospholipid alkyl chains. However, in the present case, there is also a contribution to this spectral region from the self-assembled layer of thioglucose due to the presence of $\mathrm{CH}$ and $\mathrm{CH}_{2}$ groups that also absorb IR radiation within this region (Figure 1). Therefore, in order to obtain accurate information on the conformation and orientation of the phospholipid acyl chains in the bilayer, DMPC with deuterium-substituted acyl chains was selected (Figure 1). As a result, the C-D stretching bands are shifted to lower frequencies and located in the frequency region between 2000 and $2300 \mathrm{~cm}^{-1}$, which is free of spectral overlap from all other vibrational modes and hydrogen substituted thioglucose has no C-D bands in the spectral region investigated. However, $\mathrm{d}_{54}$-DMPC has a main phase transition at $20.5{ }^{\circ} \mathrm{C}$, therefore all PM-IRRAS measurements were performed at the temperature of $18{ }^{\circ} \mathrm{C}$ to ensure that the deuterated acyl chains primarily adopt an all trans conformation of deuterated acyl chains. The spectral deconvolution of the IR absorbance bands present in the C-D stretching region is shown in Figure 4. The major bands in this region correspond to the $\mathrm{CD}_{3}$ asymmetric stretching $\left(v_{\mathrm{as}}\left(\mathrm{CD}_{3}\right)\right.$ at $\left.\sim 2216 \mathrm{~cm}^{-1}\right), \mathrm{CD}_{2}$ asymmetric stretching $\left(\mathrm{v}_{\mathrm{as}}\left(\mathrm{CD}_{2}\right)\right.$ at $\left.\sim 2196 \mathrm{~cm}^{-1}\right), \mathrm{CD}_{3}$ symmetric stretching $\left(v_{\mathrm{s}}\left(\mathrm{CD}_{3}\right)\right.$ at $\left.\sim 2154 \mathrm{~cm}^{-1}\right)$ and $\mathrm{CD}_{2}$ symmetric stretching $\left(v_{\mathrm{s}}\left(\mathrm{CD}_{2}\right)\right.$ at $\left.\sim 2091 \mathrm{~cm}^{-1}\right)$ vibrational modes. There are also two shoulder peaks on higher and lower frequency side of the $v_{\mathrm{s}}\left(\mathrm{CD}_{2}\right)$ band, which correspond to the Fermi resonance between the symmetric $\mathrm{CD}_{2}$ stretching and $\mathrm{CD}_{2}$ bending modes. ${ }^{37,38}$

Figure 5A shows the C-D spectra for a $\mathrm{d}_{54}$-DMPC bilayer transferred onto the $\mathrm{Au}(111)$ electrode modified with a $\beta$-Tg SAM at selected potentials. Additionally, the dependence of the peak center versus the applied potential is presented in the Figure 5B. The position of the $\mathrm{CD}_{2}$ vibrations provides useful information about the conformation of the acyl chains of DMPC molecules. For the all-trans conformation of the acyl chains, the $v_{\mathrm{as}}\left(\mathrm{CD}_{2}\right)$ and $v_{\mathrm{s}}\left(\mathrm{CD}_{2}\right)$ band positions are 2191 and $2088 \mathrm{~cm}^{-1}$, respectively. ${ }^{38}$ In the present case, the average frequency values of the $\mathrm{CD}_{2}$ bands are several wavenumbers higher, that is $2194 \pm 1$ and 
$2095 \pm 2 \mathrm{~cm}^{-1}$ for the $v_{\mathrm{as}}\left(\mathrm{CD}_{2}\right)$ and $v_{\mathrm{s}}\left(\mathrm{CD}_{2}\right)$ band, respectively, while the corresponding FWHM values are equal to $30.0 \pm 2.5$ and $9.0 \pm 2.0 \mathrm{~cm}^{-1}$. Such a shift in the average frequency values of the $\mathrm{CD}_{2}$ bands indicates that acyl chains are partially melted and that there is some contribution from gauche conformers. However, the frequencies presented in this work are lower than those previously reported for mixed $\mathrm{d}_{54}$-DMPC: cholesterol bilayers. ${ }^{39}$ This finding is expected since the presence of cholesterol molecules causes an increase in the overall fluidity of the film and thus an increase in the number of gauche conformers. In addition, the data presented in Figure 5B shows that there is a weak dependence of the position of these IR bands as a function of the applied potential. This dependence is more pronounced for the $v_{\mathrm{s}}\left(\mathrm{CD}_{2}\right)$ band, which displays higher frequency values at the negative potentials. This indicates that at more positive potentials the chains are slightly more ordered and that the bilayer is in a gel-like state compared to the negative potentials. Similar observation was made by Brosseau et al., who studied the three-component membranes composed of DMPC, cholesterol and GM1. ${ }^{39}$

Apart from the data concerning the conformation, the PM-IRRAS experiment also provides useful information on the orientation of the acyl chains based on the intensities of the $\mathrm{CD}_{2}$ stretching bands. The angle $(\theta)$ between the direction of the transition dipole and the surface normal can be determined using the following equation: ${ }^{40,41}$

$$
\cos ^{2} \theta=\frac{1}{3}\left(\frac{A_{(E)}}{A_{(\text {random })}}\right)
$$

where $A_{(E)}$ is the integrated intensity of the PM-IRRAS spectral band and $A_{(\text {random })}$ is the integrated intensity of the IR band for a film consisting of randomly oriented molecules. The $A_{\text {(random) }}$ can be determined from the spectra calculated from optical constants as shown by the top curve in Figure 5A. 
The calculated angles between the surface normal and the transition dipole moments of the $v_{\mathrm{s}}\left(\mathrm{CD}_{2}\right)$ and $v_{\mathrm{as}}\left(\mathrm{CD}_{2}\right)$ bands of $\mathrm{d}_{54}$-DMPC bilayer on gold electrode modified with $\beta$ - $\mathrm{Tg}$ are presented in Figure 6. The angles of the transition dipole moments for both the $v_{\mathrm{s}}\left(\mathrm{CD}_{2}\right)$ and $v_{\text {as }}\left(\mathrm{CH}_{2}\right)$ vibrations show no dependence on the applied electrode potential within experimental error. There are small differences between $\theta_{v s}$ and $\theta_{v a s}$. This suggests that there is preferential twisting of the acyl chains. The twist angle $\left(\theta_{\text {twist }}\right)$ of the trans segment of the chain is defined as the angle between the $-\mathrm{C}-\mathrm{C}-\mathrm{C}$ - plane and the plane formed by the chain axis and the surface normal. ${ }^{42,43}$ The $-\mathrm{C}-\mathrm{C}-\mathrm{C}$ - plane bisects the deuterated methylene group, the $v_{\mathrm{s}}\left(\mathrm{CD}_{2}\right)$ and $v_{\mathrm{as}}\left(\mathrm{CD}_{2}\right)$ transition dipole moments are perpendicular to one another, and $\theta_{v \mathrm{~s}}$ and $\theta_{\text {vas }}$ are defined with respect to the surface normal. As a result of these trigonometric relationships, the twist angle of the acyl chains may be found by the following $\operatorname{expression}^{42,43}$ :

$$
\theta_{\mathrm{twist}}=\tan ^{-1}\left(\frac{\cos \theta_{\mathrm{vas}}}{\cos \theta_{\mathrm{vs}}}\right)
$$

A twist angle of $45^{\circ}$ is referred to as the trivial twist angle, and corresponds to no preferential acyl chain twisting. ${ }^{42,43}$ In the present case the twist is $\sim 70^{\circ}$.

The vector of the transition dipole for the asymmetric methylene stretch lies along the plane joining the two deuterium atoms of the $\mathrm{CD}_{2}$ group, while the vector of the transition dipole for the symmetric stretch lies along the bisector of the $\mathrm{CD}_{2}$ plane. Both vectors are perpendicular to each other and are also perpendicular to the extended all-trans hydrocarbon chain. Therefore, it is possible to determine the average tilt angle of trans fragments of $\mathrm{d}_{54-}$ DMPC deuterated chain $\left(\theta_{\text {chain }}\right)$ in the bilayer using the following formula: ${ }^{44}$

$$
\cos ^{2} \theta_{v a s}+\cos ^{2} \theta_{v s}+\cos ^{2} \theta_{\text {chain }}=1
$$

where $\theta_{v a s}$ and $\theta_{v s}$ are the angles between the transition dipole moment and the surface normal for asymmetric and symmetric $\mathrm{CD}_{2}$ stretching vibrations, respectively. 
The tilt angle analysis provides information about average tilt of trans- fragments of the chains with respect to the surface normal. As an approximation we consider this number as representative for the tilt of the chains. The average tilt angles of the trans fragment of the acyl chains are plotted in Figure 7. For comparison, the tilt angle of the acyl chains of nondeuterated DMPC bilayers transferred onto bare $\mathrm{Au}(111)$ electrode by LB-LS, which were taken from a previous study, ${ }^{45}$ are also included in this figure. When the DMPC bilayer is directly deposited at the gold surface (without the underlying hydrophilic SAM), the average tilt angle of the acyl chains is approximately $25^{\circ}$ at positive potentials since the bilayer is adsorbed and remains in direct contact with the metal surface. However, when the applied potentials are stepped into negative direction, desorption of the bilayer takes place at approximately $-0.5 \mathrm{~V}$ (i.e. the bilayer becomes detached from the electrode surface). After desorption, the bilayer remains in close proximity to the gold surface and is separated from the metal by a $1-n m$ layer of solvent. ${ }^{46}$ As a result, the tilt angle decreases to an average value of $\sim 17^{\circ}$. This suggests that the bilayer adopts different structures in both the adsorbed and desorbed states. When the bilayer is adsorbed, all polar heads are in contact with the electrode surface, which limits the mobility of the inner leaflet. ${ }^{6}$ In addition, the bulky polar head group inhibits the acyl chains from packing tightly, which results in more gauche conformers and an increase the tilt angle of the acyl chains. Upon desorption, the head groups of the inner layer detach from the metal and are separated by a thin cushion of water, which exposes both leaflets of the bilayer to the electrolyte. As a result, the head groups, which no longer are in contact with metal, can be arranged in a zigzag fashion. This allows for stronger chain-chain interaction (i.e. lipid tails are able to pack more tightly), which results in an overall decrease in the tilt angle of the acyl chains. Therefore, the reported tilt angle of $17^{\circ}$ for the desorbed bilayer corresponds to a DMPC bilayer in the gel state where the polar heads 
are packed in a zigzag configuration, which has been previously observed in X-ray studies for a stack of hydrated bilayers. ${ }^{47}$

When the DMPC bilayer is separated from the metal by the self-assembled 1- $\beta-\mathrm{D}$ thioglucose layer, the tilt angles of the acyl chains of the $\mathrm{d}_{54}$-DMPC are generally smaller than those reported for the DMPC bilayer supported directly on Au(111) surface (without the $\beta$-Tg monolayer). Interestingly, the value of the tilt angle at positive potentials is equal to $18^{\circ}$, which is within the experimental error of the value observed for desorbed DMPC bilayers on the bare gold electrode (Figure 7) and in a stack of hydrated bilayers. This result implies that the presence of the hydrophilic $\beta$-Tg SAM on the $\mathrm{Au}(111)$ electrode provides a solvent-rich layer that separates the head groups of the bilayer from the metal surface at all electrode potentials. This in turn allows the polar heads of the phospholipid molecules to adopt the zigzag conformation even at positive potentials and consequently, resulted in an overall decrease in the tilt angles of the lipid tails suggesting that a more ordered layer is formed. This finding is consistent with the studies performed by Vezvaie et al., who investigated biomimetic DMPC membranes supported at a nanocavity patterned Ag electrode by means of electrochemical surface enhanced Raman scattering (EC-SERS). ${ }^{48}$ The tilt angle decreases to $\sim 14^{\circ}$ at more negative potentials where the bilayer is lifted and floating at a cushion of the electrolyte. The smaller tilt angle suggests that the acyl chains of the phospholipid molecules adopt somewhat more ordered orientation in the detached state. However, the potential dependence of the floating bilayer is very weak and the observed changes are more likely related to experimental uncertainties.

In conclusion, PM-IRRAS data indicate that the presence of self-assembled monolayer of thioglucose on the $\mathrm{Au}(111)$ electrode allows for the formation of more ordered DMPC bilayers than those deposited directly onto the gold surface. This is caused by the presence of a hydrophilic layer, which separates the bilayer from the solid support. Biomimetic 
membrane architectures such as these may be employed in future studies concerning the incorporation of proteins into the model membranes.

\section{Summary and conclusions}

PM-IRRAS was used to study the conformation and orientation of DMPC bilayers supported onto $\mathrm{Au}(111)$ electrodes modified with the self-assembled layer of $\beta$-thioglucose. Since the $\beta$-Tg molecules also absorb IR radiation, spectroscopic experiments were performed on the bilayers composed of DMPC molecules with deuterated chains in order to obtain the information on the phospholipid bilayers only. The results of the IR measurements revealed that the tilt angles of the deuterated chains in the DMPC bilayer deposited on gold electrodes pre-modified with thioglucose layers are significantly lower than those obtained previously for the DMPC bilayers transferred directly onto Au(111) electrodes. Additionally, the value of the tilt angle of $\mathrm{d}_{54}-\mathrm{DMPC}$ on $\beta-\mathrm{Tg}$ at positive potentials corresponding to the adsorbed state is equal to $\sim 18^{\circ}$, which is similar to the value obtained for the desorbed state of the h-DMPC on bare gold and to the tilt observed in a stack of hydrated bilayers. This indicates that in the presence of $\beta-\mathrm{Tg}$ on the metal surface, the phospholipid bilayer is separated from the solid support by a thin layer of water even in the adsorbed state. The tilt angles confirm that due to the presence of hydrophilic layer of thioglucose, DMPC polar heads are not in direct contact with metal surface and therefore may adopt a zigzag configuration, which allows for a smaller tilt of the acyl chains and stronger chain-chain interactions.

The results of these studies show that the application of self-assembled layer of thioglucose on the $\mathrm{Au}(111)$ electrode provides a hydrophilic environment that enables the formation of a supported biomimetic membrane where the structure and organization of the 
film are not affected by the solid substrate and thus provides a better model for a natural cell membrane. The fBLM membrane described in this study is simpler and easier to assemble than other bilayers assembled on hydrophilic spacers and discussed in the introduction. This simplicity will be significant for the future studies of antibiotic peptides incorporated into this bilayer.

\section{Acknowledgements}

This work was supported by Sinergia project no. CRSII2_154451/1 financed by the Swiss National Science Foundation and by a Discovery grant from the Natural Sciences and Engineering Research Council of Canada.

\section{References}

\footnotetext{
${ }^{1}$ Dynarowicz-Łątka, P.; Hąc-Wydro, K. Edelfosine in Membrane Environment - the Langmuir Monolayer Studies. Anti-Cancer Agents Medicin. Chem. 2014, 4, 499-508.

${ }^{2}$ Yuan, C.; Johnston, L.J. Atomic force microscopy studies of ganglioside GM1 domains in phosphatidylcholine and phosphatidylcholine/cholesterol bilayers. Biophys. J., 2001, 81, 1059-1069.

${ }^{3}$ Geraldo, V.P.N.; Pavinatto, F.J.; Nobre, T.M.; Caseli, L.; Oliveira Jr., O.N. Langmuir films containing ibuprofen and phospholipids. Chem. Phys. Letters 2013, 559, 99-106.

${ }^{4}$ Sarpietro, M.G.; Rocco, F.; Micieli, D.; Ottimo, S.; Ceruti, M.; Castelli, F. Interaction of acyclovir and its squalenoyl-acyclovir prodrug with DMPC in monolayers at the air/water interface Int. J. Pharm. 2010, 395, 167-173.

${ }^{5}$ Sandrino, B.; Tominaga, T.T.; Nobre, L.; Wrobel, E.C.; Fiorin, B.C.; de Araujo, L. Oliveira Jr., O.N.; Wohnrath, K. Correlation of [RuCl3(dppb)(VPy)] Cytotoxicity with its Effects on
} 
the Cell Membranes: An Investigation Using Langmuir Monolayers as Membrane Models. $J$. Phys. Chem. B 2014, 118, 10653-10661.

${ }^{6}$ Garcia-Araez, N.; Brosseau, C.L.; Rodriguez, P.; Lipkowski, J. Layer-by-layer PMIRRAS characterization of DMPC bilayers deposited on a Au(111) electrode surface. Langmuir 2006, 22, 10365-10371.

${ }^{7}$ Oberts B. P.; Blanchard, G. J. Formation of Air-Stable Supported Lipid Monolayers and Bilayers. Langmuir 2009, 25, 2962-2970.

${ }^{8}$ Paiva, J.G.; Paradiso, P.; Serro, A.P.; Fernandes, A.; Saramago, B. Interaction of local and general anaesthetics with liposomal membrane models: A QCM-D and DSC study. Colloids Surf. B. 2012, 95, 65-74.

${ }^{9}$ Pawłowski, J.; Juhaniewicz, J.; Guzeloglu, A.; Sęk, S. Mechanism of Lipid Vesicles Spreading and Bilayer Formation on a Au(111) Surface. Langmuir 2015, , 31, 11012-11019. ${ }^{10}$ Brand, I. Application of infrared spectroscopy for structural analysis of planar lipid bilayers under electrochemical control. Adv. Plan. Lipid Bilayers Lip., 2013, 18, 21-62.

${ }^{11}$ Juhaniewicz, J.; Sęk, S. Atomic Force Microscopy and Electrochemical Studies of Melittin Action on Lipid Bilayers Supported on Gold Electrodes. Electrochim. Acta, 2015, 162, 5361.

${ }^{12}$ Madrid, E; Horswell, S.L. Effect of Electric Field on Structure and Dynamics of Bilayers Formed From Anionic Phospholipids, Electrochim. Acta, 2014, 146, 850-860.

${ }^{13}$ Becucci, L.; Maran, F.; Guidelli, R. Probing membrane permeabilization by the antibiotic lipopeptaibol trichogin GA IV in a tethered bilayer lipid membrane Biochim. Biophys. Acta Biomembranes 2012, 1818, 1656-1662. 
${ }^{14}$ Tagami, Y.; Narita, T.; Ikigai, H.; Oishi, Y. Penetration behavior of Vibrio cholerae hemolysin into (DMPC/cholesterol) mixed monolayer. Colloids and Surfaces A 2009, 347 $225-229$.

${ }^{15}$ Nullmeier, M.; Koliwer-Brandl, H.; Kelm, S.; Zagel, P.; Koch,K. W.; Brand, I. Impact of Strong and Weak Lipid-Protein Interactions on the Structure of a Lipid Bilayer on a Gold Electrode Surface, ChemPhysChem 2011, 12, 1066 - 1079.

${ }^{16}$ Nullmeier, M.; Koliwer-Brandl, H.; Kelm, S.; Brand, I. Interaction of siglec protein with glycolipids in a lipid bilayer deposited on a gold electrode surface, J. Electroanal. Chem. 2010, 649, 177-188.

${ }^{17}$ Knoll, W.; Koper, I.; Naumann, R.; Sinner, E.K. Tethered bimolecular lipid membranes A novel model membrane platform. Electrochim. Acta 2008, 53, 6680-6689.

${ }^{18}$ Naumann, R.; Schmidt, E. K.; Jonczyk, A.; Fendler, K.; Kadenbach, B.; Liebermann, T.; Offenhausser, A.; Knoll, W. The peptide-tethered lipid membrane as a biomimetic system to incorporate cytochrome c oxidase in a functionally active form. Biosens. Bioelectron. 1999, $14,651-662$.

${ }^{19}$ Jadhav, S.R.; Rao, K.S.; Zheng, Y.; Garavito, R.M.; Worden, R.M. Voltage dependent closure of PorB class II porin from Neisseria meningitidis investigated using impedance spectroscopy in a tethered bilayer lipid membrane interface. J. Colloid Interf. Sci. 2013, 390, 211-216.

${ }^{20}$ Fragneto, G.; Charitat, T.; Daillant, J. Floating lipid bilayers: models for physics and biology. Eur Biophys J. Biophys. Lett. 2012, 41, 863-874.

${ }^{21}$ Manghi, M.; Destainville, N. Statistical Mechanics and Dynamics of Two Supported Stacked Lipid Bilayers. Langmuir 2010, 26, 4057-4068.

${ }^{22}$ Lin, J.; Szymanski, J.; Searson, P. C.; Hristova, K. Electrically Addressable, Biologically Relevant Surface-Supported Bilayers. Langmuir 2010, 26, 12054-12059. 
${ }^{23}$ Coutable, A.; Thibault, C.; Chalmeau, J.; Francois, J.M.; Vieu, C.; Noireaux, V.; Trevisiol, E. Preparation of Tethered-Lipid Bilayers on Gold Surfaces for the Incorporation of Integral Membrane Proteins Synthesized by CellFree Expression Langmuir 2014, 30, 3132-3141. ${ }^{24}$ Nikolov, V.; Lin, J.; Merzlyakov, M.; Hristova, K.; Searson, P.C. Electrical measurements of bilayer membranes formed by Langmuir-Blodgett deposition on single-crystal silicon. Langmuir 2007, 23, 13040-1304.

${ }^{25}$ Kycia, A. H.; Wang, J.; Merrill, A.R.; Lipkowski, J. Atomic Force Microscopy Studies of a Floating-Bilayer Lipid Membrane on a Au(111) Surface Modified with a Hydrophilic Monolayer. Langmuir 2011, 27, 10867-10877.

${ }^{26}$ Kycia, A. H.; Sek, S.; Su, Z.; Merrill, A. R.; Lipkowski, J. Electrochemical and STM Studies of 1-Thio-beta-D-glucose Self-Assembled on a Au(111) Electrode Surface. Langmuir 2011, 27, 13383-13389.

${ }^{27}$ Vezvaie, M.; Brosseau, C. L.; Goddard, J. D.; Lipkowski, J. SERS of beta-Thioglucose Adsorbed on Nanostructured Silver Electrodes. Chem. Phys. Chem. 2010, 11, 1460 - 1467. ${ }^{28}$ Laredo, T.; Dutcher, J. R.; Lipkowski, J. Electric Field Driven Changes of a Gramicidin Containing Lipid Bilayer Supported on a Au(111) Surface. Langmuir 2011, 27, 10072-1008. ${ }^{29}$ Chen, M.; Li, M.; Brosseau, C.L.; Lipkowski, J. AFM Studies of the Effect of Temperature and Electric Field on the Structure of a DMPC-Cholesterol Bilayer Supported on a Au(111) Electrode Surface. Langmuir 2009, 25, 1028-1037.

${ }^{30}$ Li, N.; Zamlynny, V.; Lipkowski, J.; Henglein, F. In situ IR reflectance absorption spectroscopy studies of pyridine adsorption at the $\mathrm{Au}(110)$ electrode surface. J. Electroanal. Chem. 2002, 524-525, 43-53.

${ }^{31}$ Barner, B. J.; Green, M. J.; Saez, E. I.; Corn, R. M. Polarization Modulation FourierTransform Infrared Reflectance Measurements of Thin-Films and Monolayers at Metal Surfaces Utilizing Real Time Sampling Electronics. Anal. Chem. 1991, 63, 55-60. 
${ }^{32}$ Green, M. J.; Barner M. J..; Corn, R. M. Real-Time Sampling Electronics for Double Modulation Experiments with Fourier-Transform Infrared Spectrometers. Rev. Sci. Instrum. 1991, 62, 1426-1430.

${ }^{33}$ Buffeteau, T.; Desbat, B.; Blaudez, D.; Turlet, J. Calibration procedure to derive IRRAS spectra from PM-IRRAS spectra. Appl. Spectrosc. 2000, 54, 1646-1650.

${ }^{34}$ Zamlynny, V.; Zawisza, I.; Lipkowski, J. PM FTIRRAS studies of potential-controlled transformations of a monolayer and a bilayer of 4-pentadecylpyridine, a model surfactant, adsorbed on a $\mathrm{Au}(\mathrm{III})$ electrode surface. Langmuir, 2003, 19, 132-145.

${ }^{35}$ Li, M.; Chen, M.; Sheepwash, E.;Brosseau, C. L.; Li, H.; Pettinger, B.; Gruler, H.; Lipkowski, J. AFM Studies of Solid-Supported Lipid Bilayers formed at a Au(111) Electrode Surface Using Vesicle Fusion and a Combination of Langmuir-Blodgett and LangmuirSchaefer Techniques, Langmuir 2008, 24, 10313-10323.

${ }^{36}$ Dufrene, Y. F.; Boland, T.; Schneider, J. W.; Barger, W. R.; Lee, G. U. Characterization of the physical properties of model biomembranes at the nanometer scale with the atomic force microscope. Faraday Discuss. 1998, 111, 79-94.

${ }^{37}$ Liang, C.Y.; Lytton, M.R.J. Infrared Spectra of Crystalline and Stereoregular Polymers. Polymer Sci. 1962, 61, S45-S48.

${ }^{38}$ Lee, D.C.; Durrani, A.A.; Chapman, D. A Difference Infrared Spectroscopic Study of Gramicidin-A, Alamethicin and Bacteriorhodopsin in Perdeuterated Dimyristoylphosphatidylcholine. Biochim. Biophys. Acta 1984, 769, 49-56.

${ }^{39}$ Brosseau, C. L.; Leitch, J. J.; Bin, X.; Roscoe, S. G.; Lipkowski, J. Electrochemical and PM-IRRAS a Glycolipid-Containing Biomimetic Membrane Prepared Using LangmuirBlodgett/Langmuir-Schaefer Deposition. Langmuir 2008, 24, 13058-13067. 
${ }^{40}$ Allara, D. L.; Swalen, J. D. An Infrared Reflection Spectroscopy Study of Oriented Cadmium Arachidate Monolayer Films on Evaporated Silver. J. Phys. Chem. 1982, 86, $2700-2704$.

${ }^{41}$ Allara, D. L.; Nuzzo, R. G. Spontaneously Organized Molecular Assemblies. 1. Formation, Dynamics, and Physical Properties of Normal-Alkanoic Acids Adsorbed From Solution on an Oxidized Aluminum Surface. Langmuir 1985, 1, 52-66.

${ }^{42}$ Islam, M.D.; Ren, Y.; Kato, T.Polarization modulation infrared reflection absorption spectroscopy of Gibbs monolayer at the air/water interface.Langmuir 2002, 18, 9422-9428.

${ }^{43}$ Wang, H.; Coss, C.S.; Mudalige, A.; Polt, R.L.; Pemberton, J.E. A PM-IRRAS investigation of monorhamnolipid orientation at the air-water interface.Langmuir 2013, 29, 4441-4450.

${ }^{44}$ Umemura, J.; Kamata, T.; Takenaka, T. Quantitative evaluation of molecular orientation in thin Langmuir-Blodgett films by FT-IR transmission and reflection-absorption spectroscopy. J. Phys. Chem. 1990, 94; 62-67.

45 Zawisza, I.; Bin, X.; Lipkowski, J. Potential-driven structural changes in LangmuirBlodgett DMPC bilayers determined by in situ spectroelectrochemical PM IRRAS. Langmuir 2007, 23, 5180-5194.

${ }^{46}$ Burgess, I.; Li, M.; Horswell, S. L.; Szymanski, G.; Lipkowski, J.; Majewski, J.; Satija, S. Electric field-driven transformations of a supported model biological membrane - An electrochemical and neutron reflectivity study. Biophys. J. 2004, 86, 1763-1776.

${ }^{47}$ Hauser, H.; Pascher, I.; Pearson, R. H.; Sundell, S. Preferred conformation and molecular packing of phosphatidylethanolamine and phosphatidylcholine. Biochim. Biophys. Acta 1981, $650,21-51$. 
${ }^{48}$ Vezvaie, M.; Brosseau, C. L.; Lipkowski, J. Electrochemical SERS study of a biomimetic membrane supported at a nanocavity patterned Ag electrode. Electrochim. Acta 2013, 110, $120-132$. 


\section{FIGURE CAPTIONS}

Figure 1: The molecular structures of 1-thio- $\beta$-D-glucose and $\mathrm{d}_{54}-\mathrm{DMPC}$.

Figure 2: Differential capacity curves of DMPC bilayer (red), $\beta$-Tg SAM (blue) and bare $\mathrm{Au}(111)$ (black) in $0.1 \mathrm{M} \mathrm{NaF} / \mathrm{H}_{2} \mathrm{O}$ solution.

Figure 3: Force-distance curves recorded for the DMPC bilayer deposited at the $\beta-\mathrm{Tg}$ modified gold electrode surface at $E \sim 0.2 \mathrm{~V}$ vs $\mathrm{AG} / \mathrm{AgCl}$. Inset: histogram showing the distribution of the jump-in distances observed for the force curve in $0.1 \mathrm{MNaF}$.

Figure 4: The spectral deconvolution of the C-D stretching region for a randomly oriented 5.4 $\mathrm{nm}$ thick $\mathrm{d}_{54}$-DMPC film calculated using optical constants obtained from $\mathrm{d}_{54}$-DMPC vesicles.

Figure 5: A) PM-IRRAS spectra in the C-D stretching region for the $\mathrm{d}_{54}$-DMPC bilayer deposited by LB-LS technique onto self-assembled $\beta$-thiolglucose layer on the $\mathrm{Au}(111)$ surface in a $0.1 \mathrm{M} \mathrm{NaF} / \mathrm{H}_{2} \mathrm{O}$ solution at selected potentials. The top line corresponds to the spectrum of a randomly oriented $\mathrm{d}_{54}$-DMPC film calculated from optical constants; B) Peak centers of the $v_{a s}\left(C D_{2}\right)$ (solid square) and $v_{s}\left(C_{2}\right)$ (open square) vibrations as a function of electrode potential.

Figure 6: The angle between the transition dipole moment and the surface normal for the $v_{\text {as }}\left(C_{2}\right)$ stretching (solid square) and $v_{\mathrm{s}}\left(\mathrm{CD}_{2}\right)$ stretching (open square) vibrations of the $\mathrm{d}_{54}$ DMPC acyl chains as a function of the electrode potential.

Figure 7: Comparison between the tilt angles of the acyl chains for a $\mathrm{d}_{54}$-DMPC bilayer (open squares) adsorbed onto $\beta$-thioglucose-modified $\mathrm{Au}(111)$ surface with those of a h-DMPC bilayer (solid squares) adsorbed to the bare $\mathrm{Au}(111)$ electrode surface as a function of the applied potential. The values for the h-DMPC bilayer were taken from Ref. 42. 
Figure 1

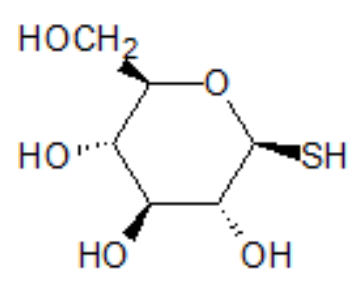

1-thio- $\beta$-D-glucose

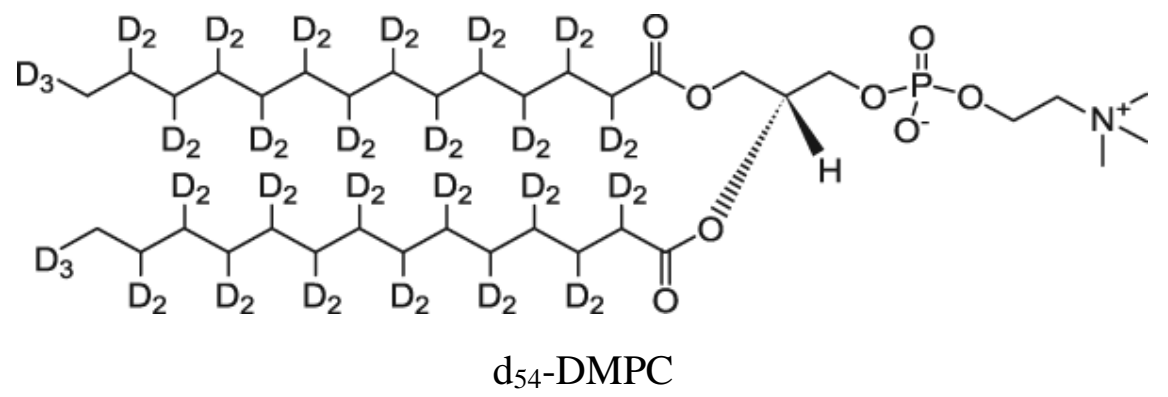


Figure 2

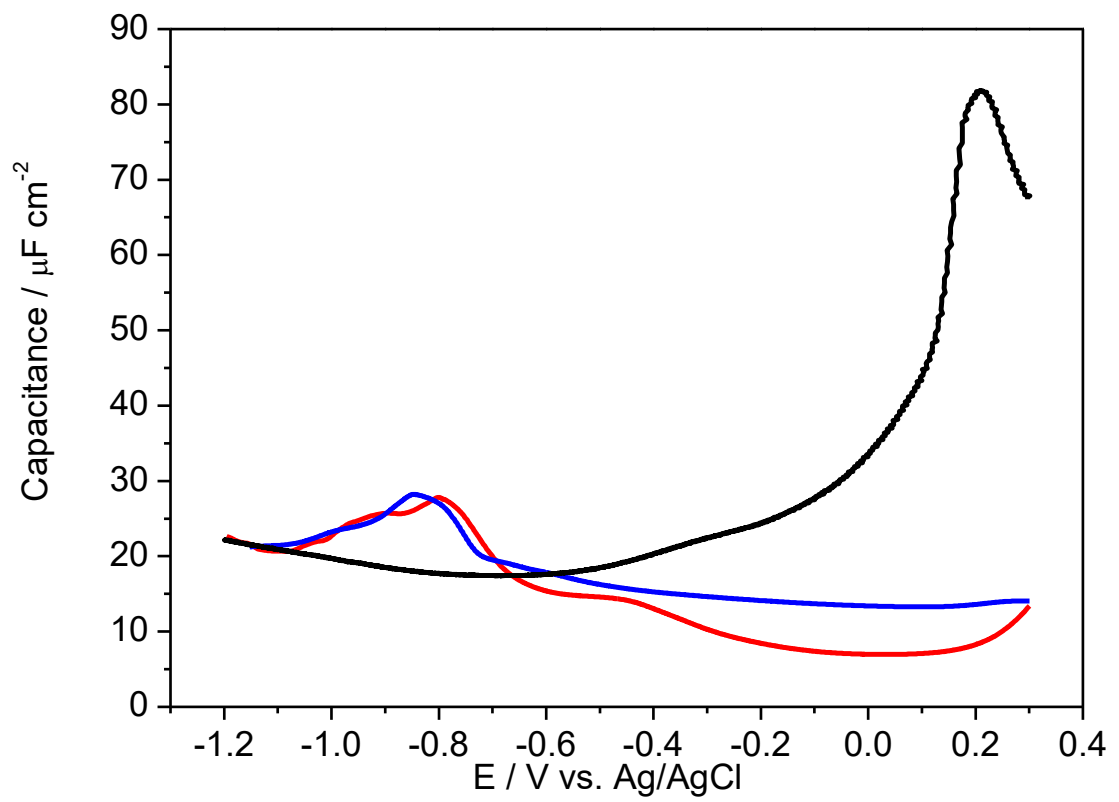


Figure 3

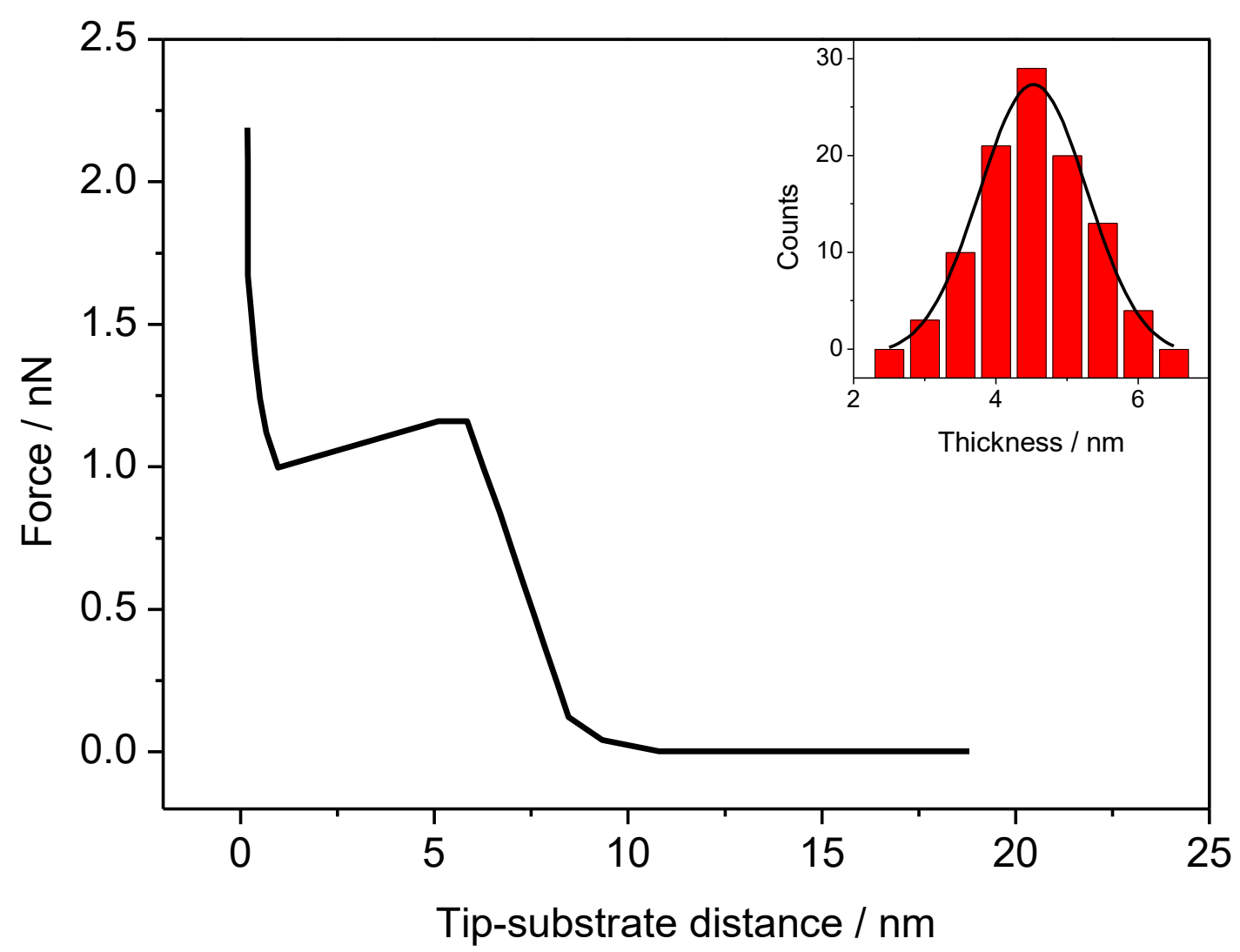


Figure 4

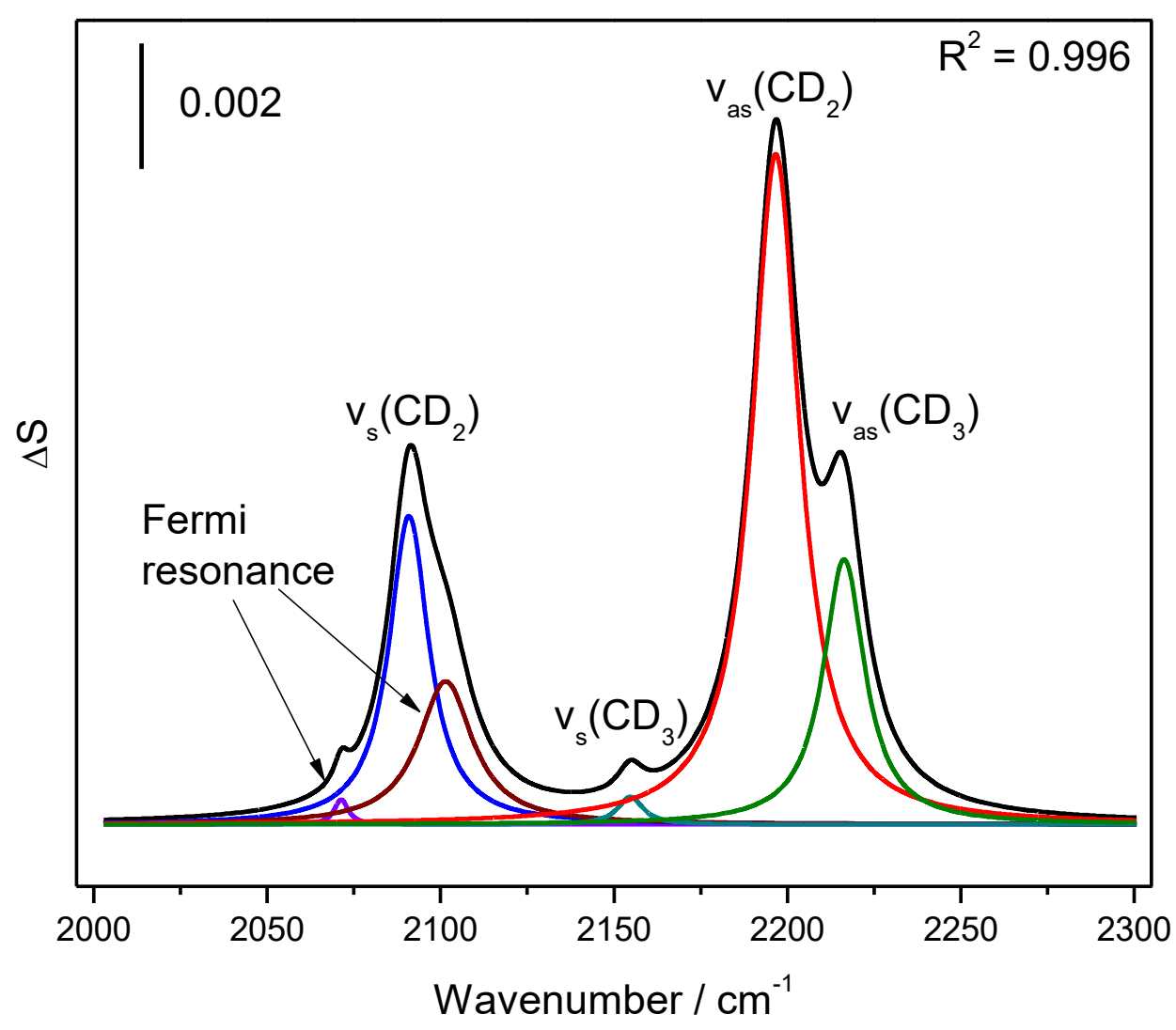


Figure 5
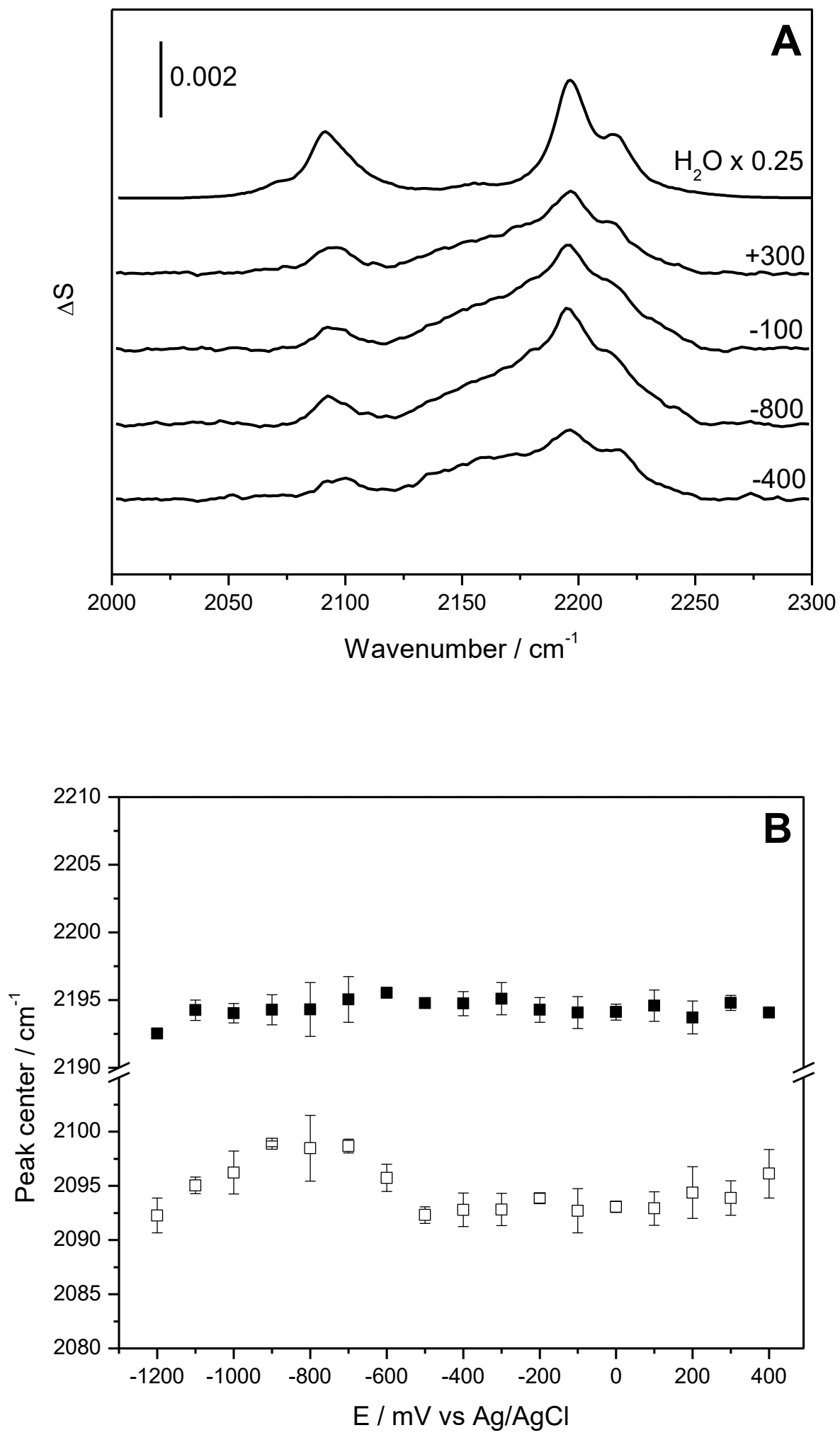
Figure 6

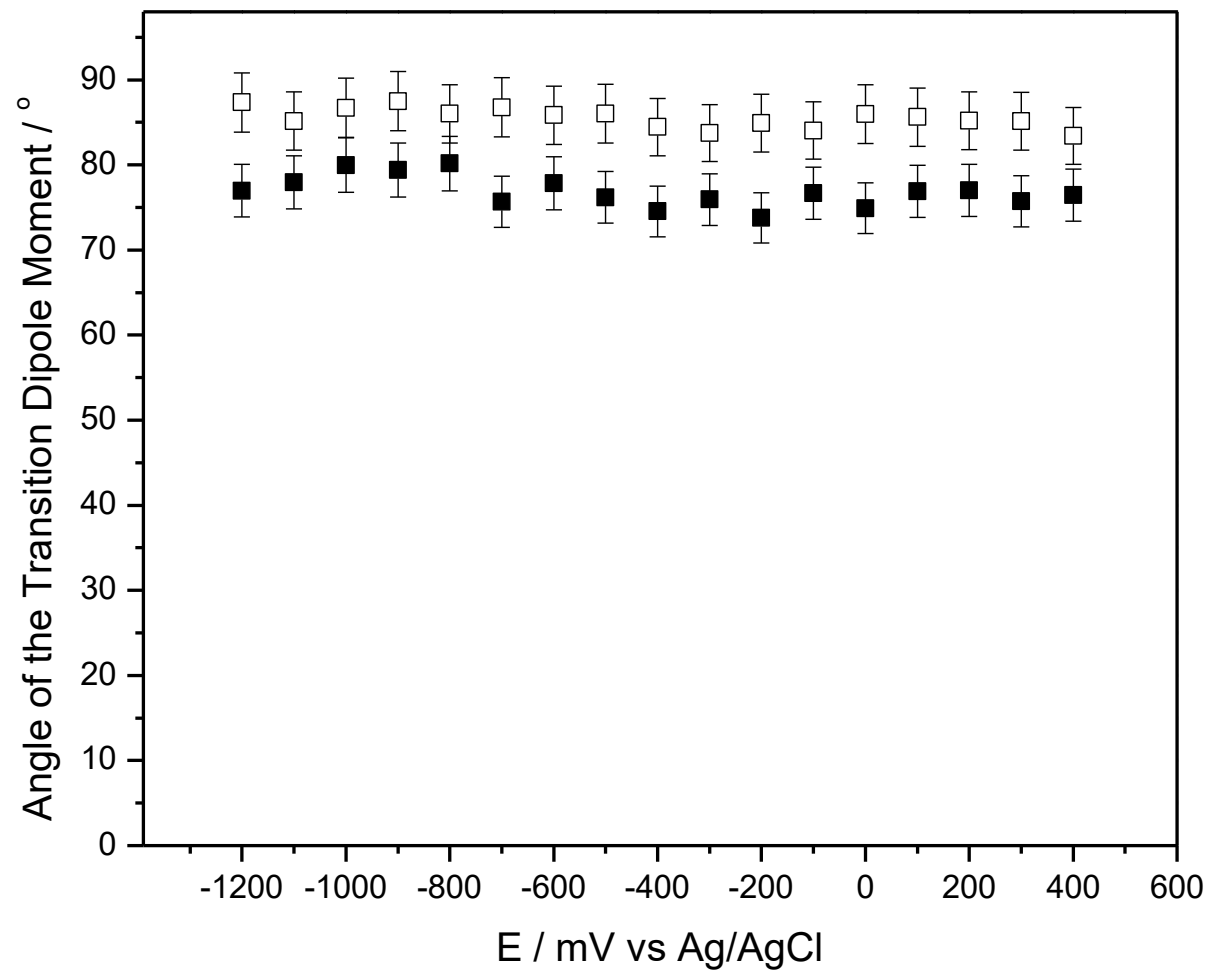


Figure 7

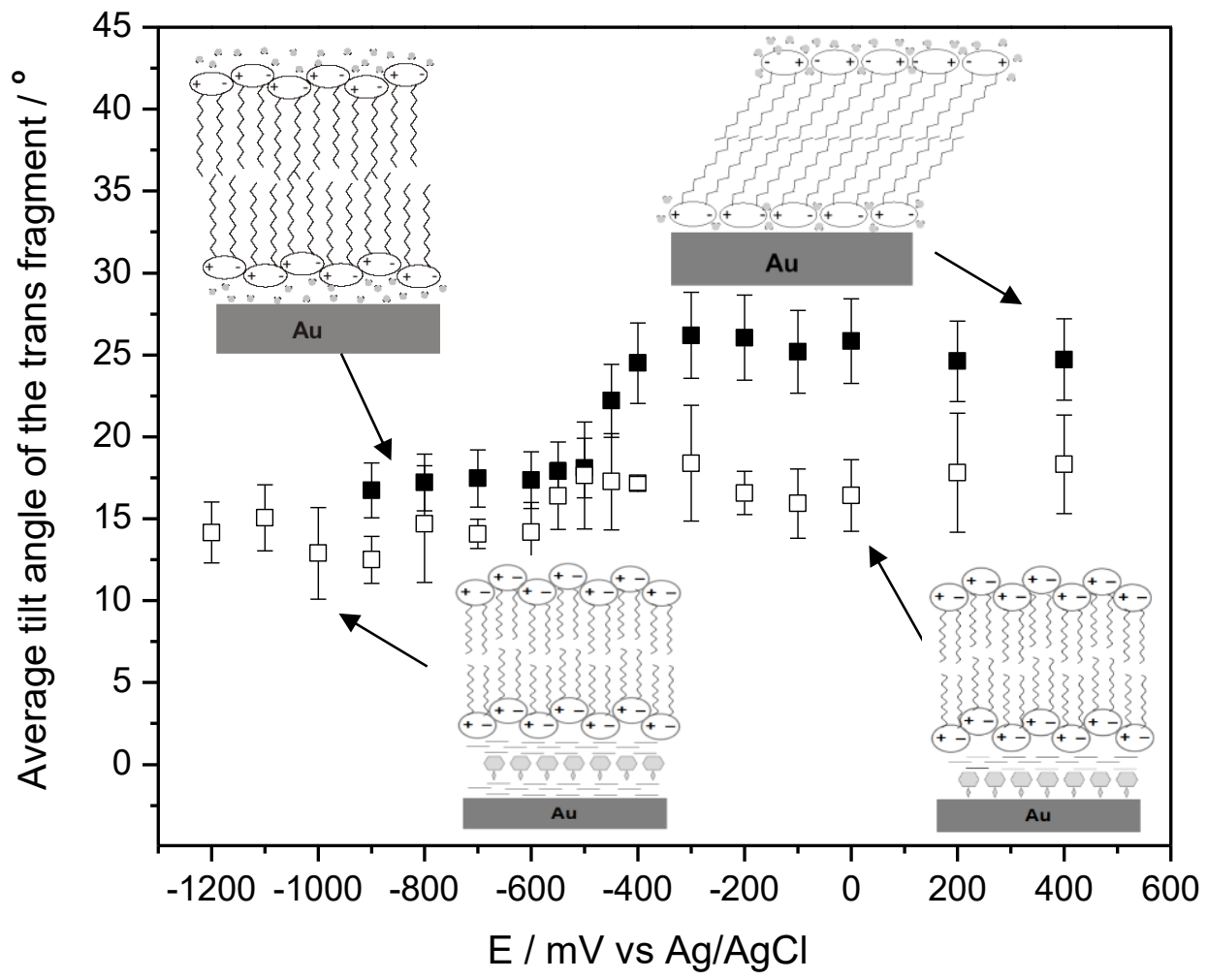


TOC
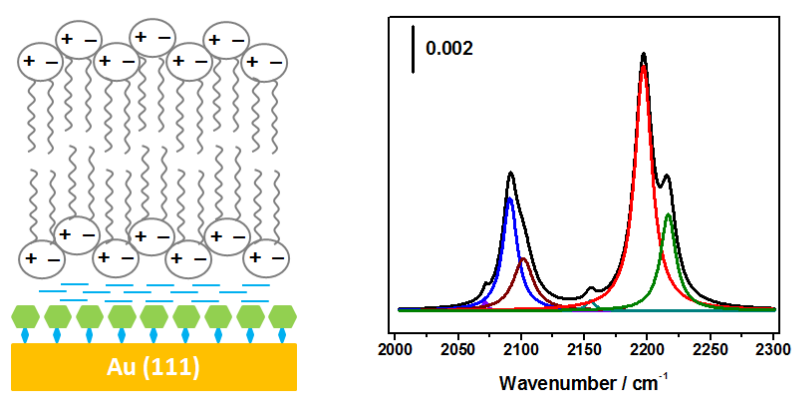